\title{
Beam dynamics and expected performance of Sweden's new storage-ring light source: MAX IV
}

\author{
S. C. Leemann, ${ }^{*}$ Å. Andersson, M. Eriksson, L.-J. Lindgren, and E. Wallén \\ MAX-lab, Lund University, S-22363 Lund, Sweden \\ J. Bengtsson \\ NSLS-II, Brookhaven National Laboratory, Upton, New York 11973, USA
}

A. Streun

SLS, Paul Scherrer Institute, CH-5232 Villigen, Switzerland

(Received 4 August 2009; published 3 December 2009)

\begin{abstract}
MAX IV will be Sweden's next-generation high-performance synchrotron radiation source. The project has recently been granted funding and construction is scheduled to begin in 2010. User operation for a broad and international user community should commence in 2015. The facility is comprised of two storage rings optimized for different wavelength ranges, a linac-based short-pulse facility and a freeelectron laser for the production of coherent radiation. The main radiation source of MAX IV will be a $528 \mathrm{~m}$ ultralow emittance storage ring operated at $3 \mathrm{GeV}$ for the generation of high-brightness hard $\mathrm{x}$ rays. This storage ring was designed to meet the requirements of state-of-the-art insertion devices which will be installed in nineteen $5 \mathrm{~m}$ long dispersion-free straight sections. The storage ring is based on a novel multibend achromat design delivering an unprecedented horizontal bare lattice emittance of $0.33 \mathrm{~nm}$ rad and a vertical emittance below the 8 pm rad diffraction limit for $1 \AA$ radiation. In this paper we present the beam dynamics considerations behind this storage-ring design and detail its expected unique performance.
\end{abstract}

DOI: $10.1103 /$ PhysRevSTAB.12.120701

PACS numbers: 29.20.db, 29.27.Bd, 29.27.Eg, 41.60.Ap

\section{INTRODUCTION}

Several high-performance third-generation synchrotron radiation sources like SLS [1], SOLEIL [2], and Diamond Light Source [3] have gone into operation in the past decade. As the technology of insertion devices (IDs) develops, requirements for synchrotron sources increase. This has sparked the design of advanced third-generation sources which push even higher brightness, submicron stability, and high constant stored current. Several such sources are presently under construction around the world: NSLS-II in the USA [4,5], PETRA III in Germany [6], and MAX IV in Sweden [7].

The MAX IV approach accounts for the fact that the different requirements of advanced synchrotron radiation sources cannot be equally fulfilled by a single machine. The required spectral range stretches from infrared to hard $\mathrm{x}$ rays while the time structure is required to satisfy both intense pulses in the fs domain as well as high average brightness in long pulses. In addition to spontaneous radiation, there is also an increasing demand for both temporally and spatially coherent radiation. A global optimization of the MAX IV facility based on this wide range of challenging demands has resulted in a solution with two separate storage rings and a linac-driven shortpulse facility which in a second stage will be upgraded with

*simon.leemann@maxlab.lu.se a free-electron laser. The two storage rings are operated at different energies to provide radiation of high brightness over a broad spectral range. The $3 \mathrm{GeV}$ linac serves as a full-energy injector for the storage rings as well as the driver of the short-pulse facility delivering intense $\mathrm{x}$-ray pulses below $100 \mathrm{fs}$ at $100 \mathrm{~Hz}$ [8]. In the first stage this will be spontaneous radiation with an upgrade to a coherent free-electron laser source based on seeding and/or cascading expected in a second stage.

The UV and soft $x$-ray storage ring will be a new $1.5 \mathrm{GeV}$ storage ring. It is similar to the existing MAX II storage ring [9] in size, but will feature a new vacuum system, an upgraded lattice, and new magnets to provide better performance for modern IDs, but also in order to extend to lower energy beam lines. Thus present-day beam lines at the $700 \mathrm{MeV}$ MAX III storage ring [10] can be moved to the new $1.5 \mathrm{GeV}$ ring. By building a new $1.5 \mathrm{GeV}$ ring rather than relocating MAX II and MAX III to the new MAX IV site, a "dark period" for UV users at MAX-lab can be avoided.

An entirely new $3 \mathrm{GeV}$ storage ring which has been optimized for hard $\mathrm{x}$ rays will complete the MAX IV facility. The lattice is based on the 20-fold seven-bend achromat described in [11]. With its unprecedented horizontal bare lattice emittance of $0.33 \mathrm{~nm}$ rad and a vertical emittance adjusted to the diffraction limit of its IDs, it is the most demanding storage ring at MAX-lab. Initial design studies [7,11-15] have been heavily revised and a 


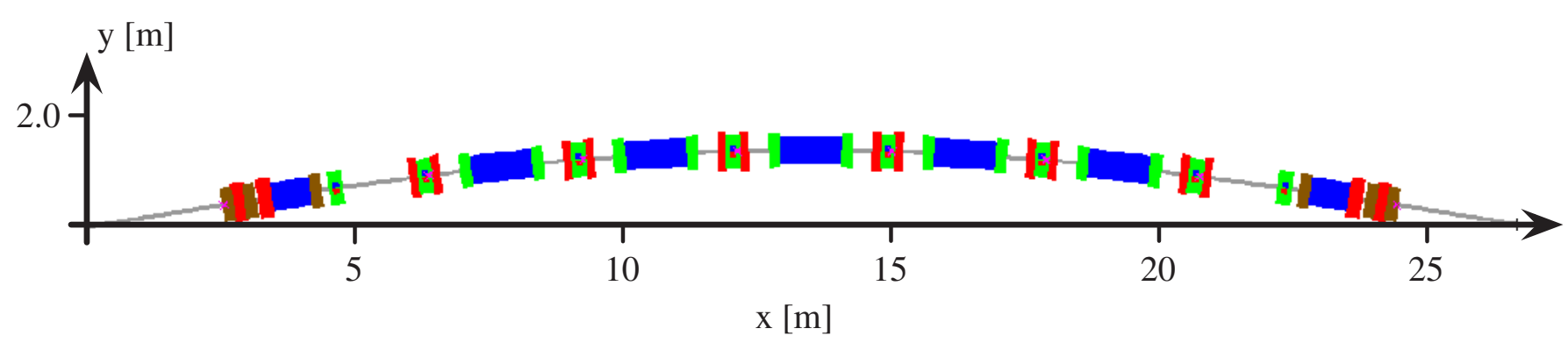

FIG. 1. (Color) Schematic of one of the 20 achromats in the $3 \mathrm{GeV}$ storage ring. Magnets indicated are gradient dipoles (blue), focusing quadrupoles (red), sextupoles (green), and octupoles (brown).

detailed design is presently being completed [16]. Construction of the facility is scheduled to begin in 2010 . User operation should commence in 2015.

This paper will highlight the MAX IV $3 \mathrm{GeV}$ storagering lattice and the beam dynamics considerations of the detailed design study. The following section presents the lattice, nonlinear optimizations and damping wigglers. Section III touches on related magnet, vacuum, and $\mathrm{rf}$ technology issues. Finally, Sec. IV demonstrates the expected dynamic performance of the lattice but also of the entire machine as a high-brightness source of synchrotron radiation. A summary and conclusions are given in Sec. V.

\section{LATTICE}

\section{A. Multibend achromat}

The figure of merit of a modern synchrotron radiation facility is a small emittance of the electron beam. The magnet lattice defines the emittance as $\varepsilon=C E^{2} / N_{d}^{3}$, where $E$ is the beam energy, $N_{d}$ is the number of dipole magnets, and $C$ is a constant given by the lattice. The bare lattice emittance is then further reduced by strong IDs. Minimizing $C$ by appropriate choice of the lattice functions is the theoretical minimum emittance design approach [17]. This approach however is hampered by problems of nonlinear optics design and high sensitivity to lattice imperfections resulting from overstrained optical functions. Instead, the MAX IV $3 \mathrm{GeV}$ storage ring is based on a very simple but robust design approach: ultralow emittance is achieved by increasing the number of dipoles $N_{d}$. In order to prevent the ring from becoming large and costly, the number of magnets is increased by shortening the magnet cells. However, shorter magnet cells require stronger focusing gradients to keep the electron optics optimized. The strong gradients required for the MAX IV $3 \mathrm{GeV}$ storage ring are achieved by a reduction of the transverse dimensions of the magnet elements (see Sec. III B for associated vacuum issues).

The storage ring is composed of 20 achromats (supercells) which each contain seven magnet cells: five unit cells plus two matching cells. The matching cells are separated from the unit cells by a $1.3 \mathrm{~m}$ short straight section where rf cavities can be installed [18]. The achromats are connected by $5 \mathrm{~m}$ long straight sections. The matching cells closely resemble the unit cell, but with only half a dipole (similar to a missing magnet dispersion suppressor). A schematic of one MAX IV $3 \mathrm{GeV}$ achromat is given in Fig. 1. The unit cells contain $3^{\circ}$ bending magnets with a gradient for vertical focusing. Focusing quadrupoles are interleaved between the dipoles [19]. The matching cells contain short $1.5^{\circ}$ gradient bends with a soft end (that is, a longitudinally varying dipole field with field strength reducing towards the long straight) to reduce synchrotron radiation heat load on the downstream ID [20]. A quadrupole doublet in the matching section allows matching of the achromat optics to the ID in the long straight section.

The optical functions for one achromat are displayed in Fig. 2 and the main parameters of the $3 \mathrm{GeV}$ storage ring are given in Table I. The strong gradient in the bending magnets yields a very compact lattice $(12.5 \mathrm{~m}$ horizontal betatron wavelength). A summary of the dipole and quadrupole strengths is given in Table II. The optics in the achromat have been tuned in such a way that the vertical beam size in the straight section is below the diffraction limit even for modest values of coupling. At a radiated wavelength of $\lambda_{\text {rad }}=1 \AA$, the photon diffraction limit is $\varepsilon_{\mathrm{rad}}=\lambda_{\mathrm{rad}} / 4 \pi=8 \mathrm{pm}$ rad. This is $2.4 \%$ of the horizontal

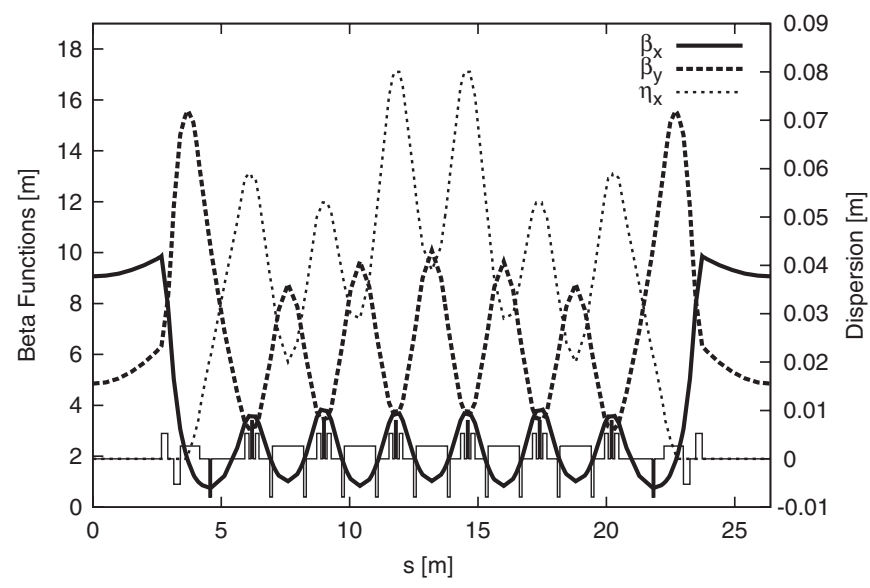

FIG. 2. Beta functions $\beta_{x}, \beta_{y}$ and dispersion $\eta_{x}$ for one achromat of the $3 \mathrm{GeV}$ storage ring. The position of the dipoles, quadrupoles, and sextupoles are indicated at the bottom. 
TABLE I. Parameters for the MAX IV $3 \mathrm{GeV}$ storage ring. Permanent-magnet damping wigglers (PMDWs) are used to further reduce the storage-ring emittance (see Sec. IIC).

\begin{tabular}{lc}
\hline \hline Energy [GeV] & 3.0 \\
Main radio frequency [MHz] & 99.931 \\
Harmonic number & 176 \\
Circulating current [mA] & 500 \\
Circumference [m] & 528 \\
Number of achromats & 20 \\
Number of long straight sections available for IDs & 19 \\
Betatron tunes (horizontal/vertical) & $42.20 / 14.28$ \\
Natural chromaticities (horizontal/vertical) & $-49.8 /-43.9$ \\
Corrected chromaticities (horizontal/vertical) & $+1.0 /+1.0$ \\
Momentum compaction factor & $3.07 \times 10^{-4}$ \\
Horizontal damping partition $J_{x}$ & 1.86 \\
Horizontal emittance (bare lattice) [nm rad] & 0.326 \\
Horizontal emittance (with four PMDWs) [nm rad] & 0.263 \\
Radiation losses per turn (bare lattice) [keV] & 360.0 \\
Radiation losses per turn (with four PMDWs) [keV] & 572.1 \\
Natural energy spread & $0.077 \%$ \\
Energy spread (with four PMDWs) & $0.096 \%$ \\
Required dynamic acceptance (horizontal/vertical) [mm mrad] & $7.1 / 1.3$ \\
Required lattice momentum acceptance & $\pm 4.5 \%$ \\
\hline \hline
\end{tabular}

bare lattice emittance and corresponds to a vertical beam size of $6 \mu \mathrm{m}$ at the center of the straight section. However, for longer wavelength beam lines, a larger vertical beam size would be sufficient to meet the diffraction limit. Because of intrabeam scattering and lifetime concerns (see Sec. IVE), it is not advisable to choose a vertical beam size below the diffraction limit. Therefore it is foreseen to include a set of geometric and chromatic skew quadrupoles in the lattice in order to gain tight vertical beam size control. Betatron coupling leading to spurious vertical dispersion will be suppressed so the vertical beam size meets the diffraction limit in the IDs delivering the hardest radiation. The vertical beam size in the achromats can then be increased in order to retain good lifetime by local control of the vertical dispersion $\left(h_{00101}\right)$ without driving global linear coupling $\left(h_{10100}\right.$ and $\left.h_{10010}\right)$ [21].

\section{B. Nonlinear optics and higher-order correction}

Although the overall optics are relaxed (considering the ratio $\xi_{x} / \nu_{x} \approx-1.2$ ), the compact lattice is of strong focusing nature leading to a large negative natural chromaticity. Chromatic sextupoles are required in order to reduce this high natural chromaticity. The nonlinearities of the lattice then have to be corrected to retain sufficient dynamic aperture. Because of the modest dispersion in the achromat, the chromatic sextupoles are strong. It was originally foreseen to integrate the focusing sextupole into the focusing quadrupoles between the unit cell dipoles. This idea was later dropped in order to retain full tuning capability and reduce nonlinearities arising from the long sextupole magnets. Instead, the focusing quadrupoles between the unit cell dipoles were split up and a short sextupole inserted in the center (see Sec. III A). This puts the

TABLE II. Dipole field and quadrupole gradients for different magnet families in the MAX IV $3 \mathrm{GeV}$ storage ring (bare lattice). For the dipoles, the length given corresponds to the iron length; for the quadrupoles the hard-edge magnet length is specified. The quadrupole gradient of the matching cell dipole specified here corresponds to the peak strength; the dipole gradient in the soft-end dipole varies longitudinally in the same way as the dipole field.

\begin{tabular}{lccc}
\hline \hline & $\begin{array}{c}\text { Length } \\
{[\mathrm{m}]}\end{array}$ & $\begin{array}{c}\text { Dipole } \\
\text { peak field [T] }\end{array}$ & $\begin{array}{c}\text { Quadrupole } \\
\text { gradient [T/m] }\end{array}$ \\
\hline Dipole (unit cell) & 0.974 & 0.524 & -8.69 \\
Soft-end dipole (matching cell) & 0.554 & 0.524 & -8.69 \\
Focusing quadrupole (unit cell) & $2 \times 0.15$ & $\ldots$ & 40.39 \\
Focusing quadrupole (facing matching cell) & $2 \times 0.15$ & $\ldots$ & 37.80 \\
Final focusing quadrupole (matching cell) & 0.25 & $\ldots$ & 35.34 \\
Final defocusing quadrupole (matching cell) & 0.25 & $\ldots$ & -22.40 \\
\hline \hline
\end{tabular}


TABLE III. Sextupole and octupole gradients for different magnet families in the MAX IV $3 \mathrm{GeV}$ storage ring (bare lattice). The specified length corresponds to the hard-edge field length.

\begin{tabular}{lccc}
\hline \hline & $\begin{array}{c}\text { Length } \\
{[\mathrm{m}]}\end{array}$ & $\begin{array}{c}\text { Sextupole } \\
\text { gradient }\left[\mathrm{T} / \mathrm{m}^{2}\right]\end{array}$ & $\begin{array}{c}\text { Octupole } \\
\text { gradient }\left[\mathrm{T} / \mathrm{m}^{3}\right]\end{array}$ \\
\hline Focusing sextupoles (unit cell) & 0.1 & $2148 / 1700 / 1600$ & $\ldots$ \\
Defocusing sextupole (unit cell) & 0.1 & -1179 & $\ldots$ \\
Defocusing sextupole (matching cell) & 0.1 & -1340 & $\ldots$ \\
Focusing octupole (matching cell) & 0.1 & $\ldots$ & 21814 \\
Defocusing octupoles (matching cell) & 0.1 & $\ldots$ & $-13143 /-6886$ \\
\hline \hline
\end{tabular}

sextupoles at local dispersion maxima which relaxes magnet requirements.

In addition to these focusing sextupoles, defocusing sextupoles have been installed in pairs flanking the unit cell dipoles and on the high-field end of the matching cell dipole. Because of the large number of installed sextupoles and the small magnet apertures (25 $\mathrm{mm}$ diameter), the sextupoles can be kept short. The OPA code [22] was used to optimize the sextupole settings: in addition to correcting the linear chromaticity to $\xi_{x, y}=+1.0$ [23] in both planes (driven by terms $h_{11001}$ and $h_{00111}$ ), two firstorder chromatic $\left(h_{20001}\right.$ and $\left.h_{00201}\right)$ and five first-order geometric terms $\left(h_{21000}, h_{10110}, h_{30000}, h_{10020}, h_{10200}\right)$ of the sextupole Hamiltonian were minimized along with the quadratic chromaticities $\left(\xi_{x}^{(2)}, \xi_{y}^{(2)}\right)$ and amplitudedependent tune shifts $\left(\partial \nu_{x} / \partial J_{x}, \partial \nu_{y} / \partial J_{y}, \partial \nu_{x} / \partial J_{y}=\right.$ $\left.\partial \nu_{y} / \partial J_{x}\right)$ [24,25]. Resulting amplitude-dependent tune shifts and chromatic behavior were verified with TRACY-3 [26] for the bare lattice and the lattice with IDs.

The high periodicity of the lattice reduces the number of allowed resonances. Although the choice of working point keeps the tunes clear of potentially limiting resonances, the number of sextupole families used to minimize the abovementioned terms was insufficient. Amplitude-dependent tune shifts remained too large leading to reduced dynamic aperture. However, since amplitude-dependent tune shifts are a second-order effect from the sextupole Hamiltonian, it is clear that sextupoles are inefficient at fighting them. Instead, three dedicated harmonic octupole families were added to the matching cell at positions with optimum beta function ratios $[27,28]$.

These three octupole families were then used to effectively fight the three Hamiltonian driving terms for amplitude-dependent tune shift: $h_{22000} \rightarrow \partial \nu_{x} / \partial J_{x}$, $h_{00220} \rightarrow \partial \nu_{y} / \partial J_{y}, \quad h_{11110} \rightarrow \partial \nu_{x} / \partial J_{y}=\partial \nu_{y} / \partial J_{x}$. Accordingly, the sextupoles could then be reoptimized to fight only first-order sextupole terms and adjust chromatic tune shifts. By properly tuning the cubic chromaticities $\left(\xi_{x}^{(3)}, \xi_{y}^{(3)}\right)$ and minimizing the quadratic chromaticities $\left(h_{11002} \rightarrow \xi_{x}^{(2)}, h_{00112} \rightarrow \xi_{y}^{(2)}\right)$, the chromatic tune shift can be "wrapped up" around the working point keeping the chromatic tune footprint very compact (cf. Fig. 13). A summary of the sextupole and octupole parameters is given in Table. III. An in-depth comparison of the MAX IV $3 \mathrm{GeV}$ storage-ring lattice and its performance with and without higher-order octupole correction is currently being prepared for publication in [28].

Because of the large beta function ratios at the chosen octupole locations, the required octupole strengths are low and the magnets can be kept short and simple. With the proper octupole tuning, the amplitude-dependent tune shifts can be reduced to very small values within the physical aperture (see Fig. 3). As a result of the sextupole reoptimization, the chromatic tune footprint has been minimized and the overall sextupole strength reduced (see Fig. 4).
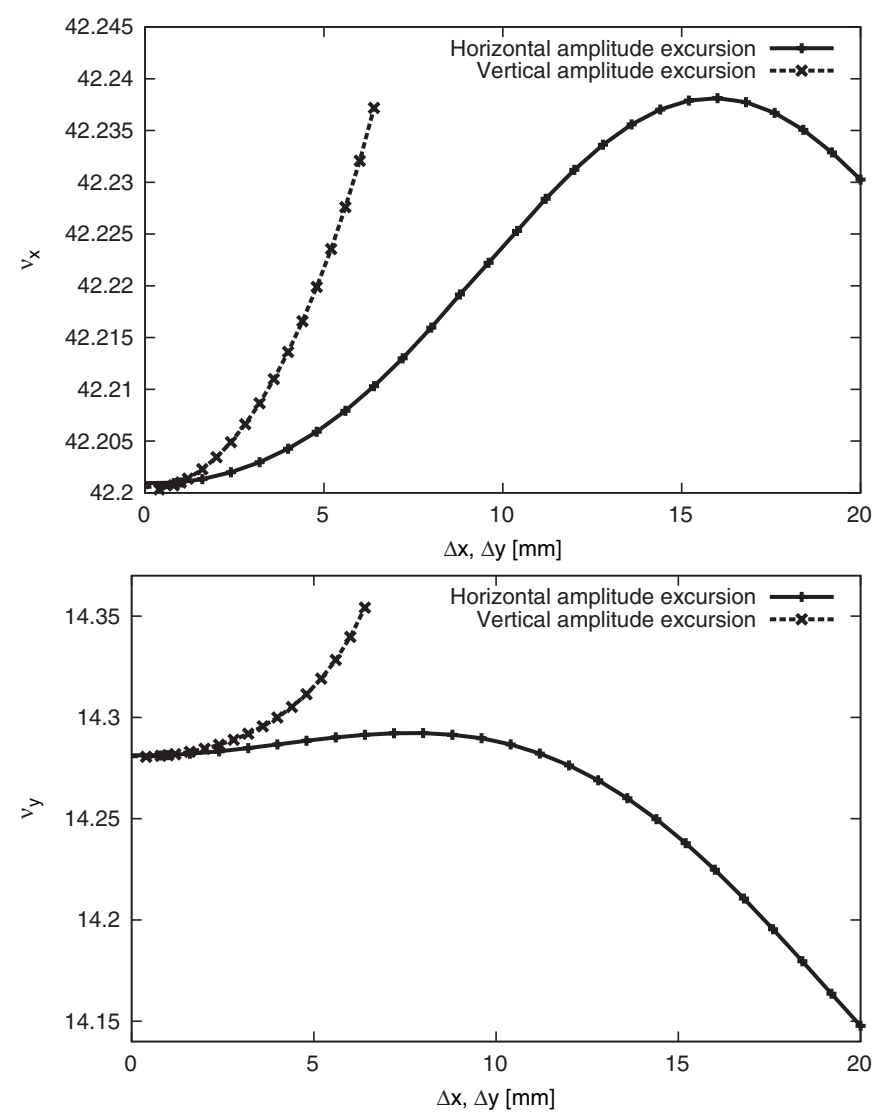

FIG. 3. Amplitude-dependent tune shifts for the $3 \mathrm{GeV}$ storage-ring bare lattice. Sextupoles and octupoles have been included in this calculation. 


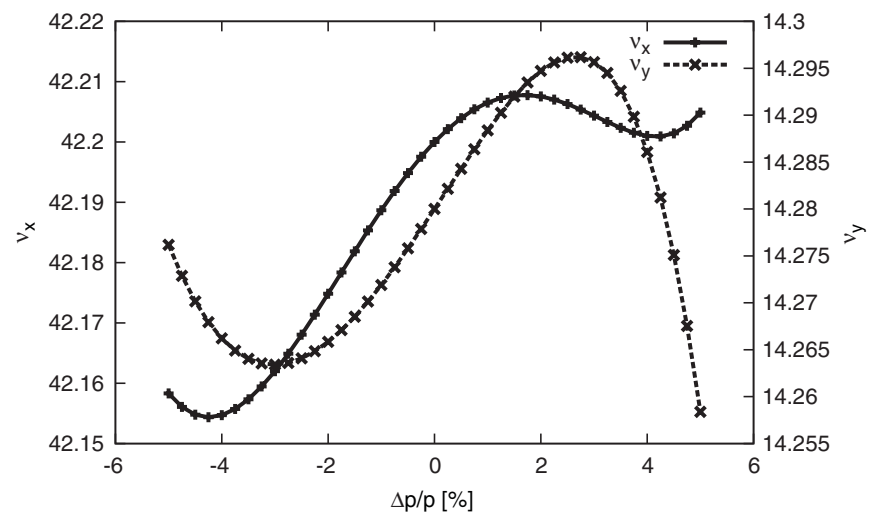

FIG. 4. Chromaticity for the $3 \mathrm{GeV}$ storage-ring bare lattice. Sextupoles and octupoles have been included in this calculation. The linear chromaticity has been corrected to +1.0 in both planes.

The resulting nonlinear optics settings also ensure that the two synchrobetatron terms $h_{20001}$ and $h_{00201}$ are kept as small as possible. This is crucial for good longitudinal acceptance (see Sec. IV B) because these terms drive the momentum-dependent beat of the beta functions $\left(d \beta_{x} / d \delta\right.$, $d \beta_{y} / d \delta$ ). Dependence of the beta functions (at the center of the long straight section) on momentum deviation is shown in Fig. 5.

\section{Damping wigglers}

Damping wigglers will be used to further reduce the bare lattice emittance and damping time. Two cases were studied so far. The first option makes use of two superconducting damping wigglers installed in opposite straight sections. Each of these devices is $1.5 \mathrm{~m}$ long and has a peak magnetic field of 3.5 T. An alternative option foresees the

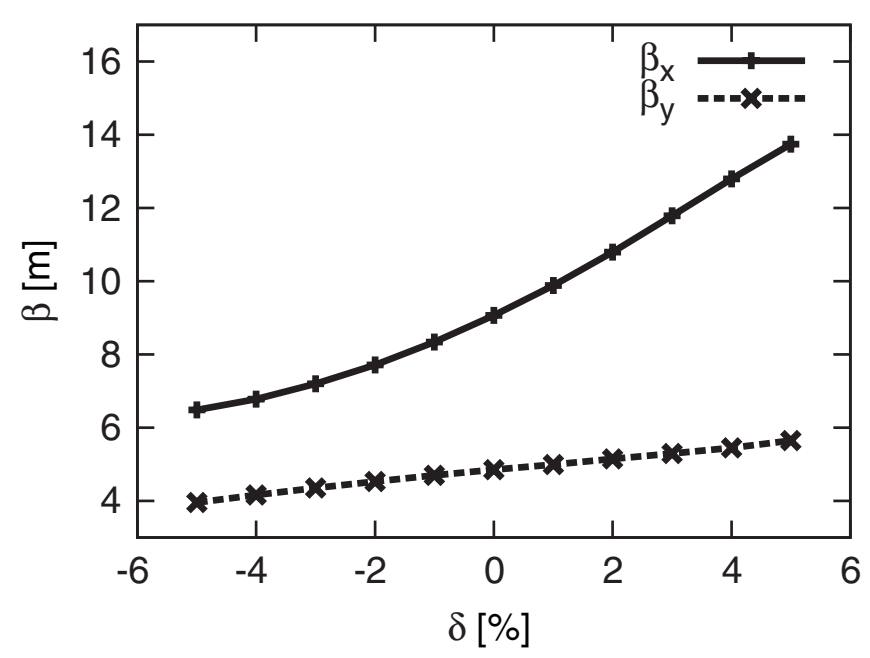

FIG. 5. Dependence of the beta functions (at the center of the long straight section) on momentum deviation for the bare lattice. Sextupoles and octupoles have been included in this calculation.
TABLE IV. Parameters of the permanent-magnet damping wigglers.

\begin{tabular}{lc}
\hline \hline Length [m] & 2.0 \\
Period length [mm] & 80 \\
Peak magnetic field strength [T] & 2.22 \\
Full gap height [mm] & 4 \\
Radiated energy per turn [keV] & 53 \\
\hline \hline
\end{tabular}

installation of two or four normal-conducting permanentmagnet damping wigglers (PMDWs) in several straight sections. These devices will be $2 \mathrm{~m}$ long and have a peak magnetic field of $2.22 \mathrm{~T}$ (see Table IV). If four of these devices are installed in the MAX IV $3 \mathrm{GeV}$ storage ring, the horizontal emittance can be reduced from 0.33 to $0.26 \mathrm{~nm} \mathrm{rad}$. The radiated energy per turn is increased from 360 to $572 \mathrm{keV}$ while the damping time is reduced by $24 \%$.

Because of their strong vertical focusing effect, the damping wigglers have to be compensated in order to ensure optimum dynamic performance. This will be done in two steps. First, the final quadrupoles in the matching sections flanking the damping wigglers are adjusted to reduce the vertical beta function in the straight section making the beam less sensitive to the vertical focusing of the wiggler. In principle, this local compensation could be achieved in the opposite manner by reducing the vertical focusing at the entry to the straight to compensate for the additional vertical focusing of the wiggler. This however has the drawback of increasing the vertical beam size within the IDs. We therefore chose to compensate by increasing the vertical focusing in the straight even though this comes at the cost of a larger overall phase advance. In a second step, the main focusing quadrupole family and the defocusing pole strips in the dipoles are adjusted to restore the global working point. Studies have shown that this procedure does not necessarily require retuning the sextupole and octupole optics [16].

It is currently under investigation if such compensation should also be implemented in a feed-forward scheme for strong in-vacuum undulators with variable gap settings. The great advantage of such a combined local/global compensation for IDs is that the optical functions and tunes in the entire machine are kept as close as possible to their bare lattice design values for which the nonlinear optics have been optimized. The result is that dynamic performance should remain high regardless of ID gap settings since the working point is always restored and tune shifts away from it are kept small.

\section{TECHNOLOGY}

\section{A. Magnets}

The magnets for the MAX IV $3 \mathrm{GeV}$ storage ring are required to be very compact. Together with the very low 


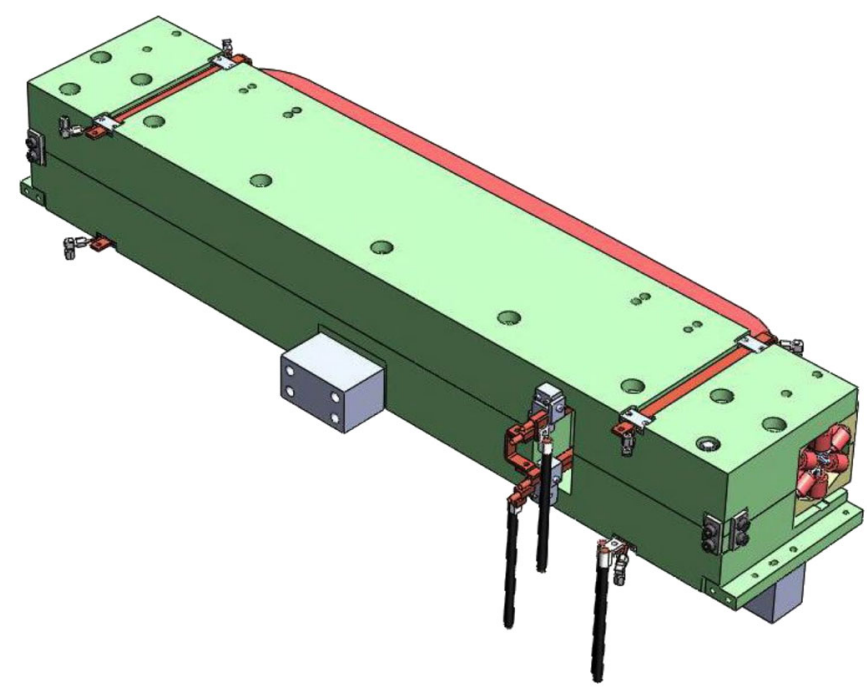

FIG. 6. (Color) Illustration of the unit cell dipole magnet block. The common iron block integrates the unit cell dipole and the two flanking defocusing sextupoles. Further integration of the unit cell focusing quadrupoles and focusing sextupole is presently under consideration.

emittance of the beam, this calls for very strong multipole magnets. The foreseen solution is to use magnets with very small apertures and a narrow vacuum chamber. The dipoles are C-type with a gradient; the gap therefore varies around the center value of $28 \mathrm{~mm}$. The quadrupole, sextupole, and octupole magnets have full apertures of $25 \mathrm{~mm}$. The good field region (defined as the area where the main field component is within $\pm 0.02 \%$ of the design field strength) of these magnets extends to no less than $\pm 10 \mathrm{~mm}$ in the horizontal plane.

As already successfully demonstrated in MAX III [10], several magnets will be machined into a common iron block resulting in an integrated girder-magnet design. This inexpensive design approach has the advantage that alignment errors can be reduced to the level of machining accuracy (on the order of $10 \mu \mathrm{m}$ ). For the MAX IV $3 \mathrm{GeV}$ storage ring it is foreseen to integrate the magnets in the unit cells and the magnets in the matching cells. For the unit cell this calls for integration of the two defocusing sextupoles together with the $3^{\circ}$ unit cell dipoles (displayed in Fig. 6), possibly including the focusing quadrupoles and focusing sextupoles between the unit cells. In the matching cell all the magnets between the long straight and short straight will be integrated: the $1.5^{\circ}$ soft-end dipole, the final focusing quadrupole doublet, three octupoles, and two beam position monitor (BPM)/corrector pairs (see Fig. 7).

The dipole magnet blocks will be installed on massive concrete supports at low height. In between these structures, quadrupole magnet blocks are installed on rigid bridge girders attached on both sides to the massive concrete supports. Alignment of the integrated magnet blocks on the concrete support will be done with adjustment screws similar to MAX III; beam-based alignment of the magnet cells will be performed using an established scheme based on corrector magnet strengths for the corrected orbit $[10,29]$. The benefit of using small and therefore light magnets on massive but low support blocks is that the eigenfrequencies of the magnets are pushed beyond $100 \mathrm{~Hz}$ [16]. This is very important for the MAX IV $3 \mathrm{GeV}$ storage ring since the tolerances for beam vibrations are very tight given the ultralow beam size (a vertical beam size on the order of $3 \mu \mathrm{m}$ at the ID center calls for a vertical beam stability of $\approx 300 \mathrm{~nm}$ in the straight sections).

Pole face strips will be included in the dipoles to allow for adjustments of the defocusing quadrupole strength. The focusing sextupoles as well as the final defocusing sextupole family are foreseen to carry additional windings so that horizontal and vertical orbit correction can be integrated (as done at SLS). In addition, there will be additional extra windings on the defocusing sextupoles for use

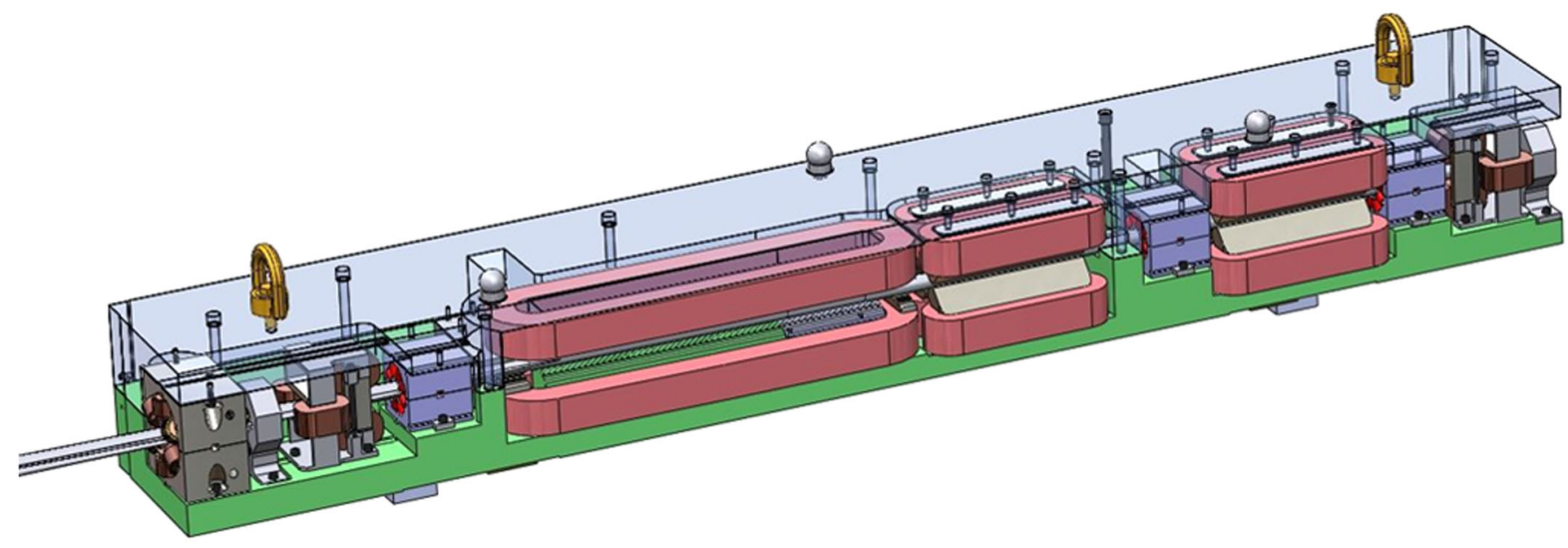

FIG. 7. (Color) Illustration of the matching cell magnet block. The common block integrates the $1.5^{\circ}$ soft-end dipole, the final focusing quadrupole doublet, three octupoles, and two BPM/corrector pairs. 
as either auxiliary sextupoles (since all regular sextupoles are powered in families) or as skew quadrupoles. Additional windings on the two octupole families installed closest to the ID could be used as nondispersive skew quads.

\section{B. Vacuum system}

The small magnet apertures require a narrow vacuum chamber. Such vacuum systems are usually plagued by poor vacuum conductance. This problem has been solved for narrow-gap ID vacuum chambers by the introduction of nonevaporable getter (NEG) coating [30]. For the MAX IV $3 \mathrm{GeV}$ storage ring, it is foreseen to extend this technique to the entire storage-ring vacuum chamber. First tests using NEG-coated copper tubes as dipole vacuum chambers in MAX II have shown promising results [31,32].

It has therefore been decided to use a circular NEGcoated copper tube with an outer diameter of $24 \mathrm{~mm}$ within the storage-ring achromats. In this way lumped absorbers can be avoided as synchrotron radiation is distributed along the tube yielding a lower power density. Small discrete ion pumps will be installed to pump rest gas along with dedicated roughing pumps. Short tapering sections in the straights will make the transition from the circular beam pipe to either the narrow-gap chamber or the ID chamber in case of in-vacuum devices. Heating for bakeout and reactivation of the NEG can take place in situ if baking tape is applied around the outside of the chamber as has been successfully demonstrated at SOLEIL [33].

\section{C. rf system}

Since the MAX IV facility has its own dedicated shortpulse facility, the storage rings can be operated at low radio frequencies with long bunches. Long electron bunches have the advantage of a narrow power spectrum which counteracts the resistive wall instability and thus reduces the need to run at large positive chromaticities restricting the energy acceptance.

It is therefore foreseen to use $100 \mathrm{MHz}$ rf cavities in the MAX IV $3 \mathrm{GeV}$ storage ring. There is a lot of experience at MAX-lab with this cavity type [34]. Higher-order modes are pushed to relatively high frequencies, where their influence is diminished due to a poor form factor. Six such cavities operated at $250 \mathrm{kV}$ gap voltage will ensure $5.3 \%$ rf momentum acceptance for a machine with four PMDWs and $4.0 \%$ rf momentum acceptance for a machine with four PMDWs and ten in-vacuum undulators (IVUs) [35].

The cavities are installed in the short straight sections keeping all but one long straight section (injection straight) free for IDs. Cavities are joined in pairs with $3 \mathrm{~dB}$ hybrids and connected to four $50 \mathrm{~kW}$ tetrode amplifiers. This scheme has the advantage that it reduces reflected power to the amplifier without the need for expensive circulators. At the same time, power regulation can be achieved by phasing rather than by changing the power level of the amplifier.

In addition to the main rf cavities, three passive thirdharmonic Landau cavities will be installed to further lengthen the bunches and improve lifetime. With such a double rf system the bunch length can be increased from $10 \mathrm{~mm}$ for the bare lattice (or $12 \mathrm{~mm}$ in the case of the machine with four PMDWs and ten IVUs) by a factor $4-5$ [16].

\section{LATTICE AND MACHINE PERFORMANCE}

\section{A. Dynamic aperture}

Achieving sufficient dynamic aperture is crucial for good Touschek lifetime and injection efficiency. We foresee injection with a localized four-kicker bump in the $5 \mathrm{~m}$ long straight section. The stored beam is bumped $8 \mathrm{~mm}$ toward the $2.5 \mathrm{~mm}$ thick Lambertson septum [16]. At the septum, separation between the stored beam and the injected beam is $5.5 \mathrm{~mm}$. To this we add a safety margin and set the horizontal dynamic aperture requirement at $\pm 8 \mathrm{~mm}$ in the straight section (corresponding to a horizontal acceptance requirement of $7.1 \mathrm{~mm}$ mrad). In the vertical plane dynamic aperture requirements of a modern synchrotron radiation source are modest due to successful operation of minigap in-vacuum IDs. With such half-gaps on the order of $2 \mathrm{~mm}$ and typically $\beta_{y}=3 \mathrm{~m}$ at the end of the IDs, a vertical acceptance requirement of $1.3 \mathrm{~mm} \mathrm{mrad}$ should be sufficient.

Dynamic aperture for the MAX IV $3 \mathrm{GeV}$ storage ring has been calculated with both OPA and TRACY-3. The PMDWs have been modeled in TRACY-3 with both the wiggler model as well as with kick maps generated by RADIA [36]. Very good agreement was observed between results obtained with the OPA undulator model, the TRACY-3 wiggler model, and kick maps inserted in TRACY-3. Results for the center of the $5 \mathrm{~m}$ straight section indicate sufficient dynamic aperture in both planes for on- and off-momentum particles as shown in Fig. 8. If the physical boundaries are included in the lattice file, the dynamic aperture extends to these boundaries confirming that nonlinearities are very small within the physical aperture.

Dynamic aperture was also calculated for a machine with imperfections. In a first study, ignoring girder correlations, quadrupole and sextupole magnets were assumed misaligned by $50 \mu \mathrm{m}$ in both the horizontal and the vertical direction as well as rolled by $0.2 \mathrm{mrad}$ (these are rms misalignments with a cutoff at $2 \sigma$ ). In addition, higherorder multipole errors were added to all quadrupoles and sextupoles. A tentative BPM/corrector layout with 10 BPMs and corrector pairs in each achromat was then used to simulate orbit correction with TRACY-3 and to obtain statistics (different random seeds). The resulting maximum required corrector strength was below $0.2 \mathrm{mrad}$ and maximum offsets for the closed orbit remained below $70 \mu \mathrm{m}$ in both planes. For each error seed 


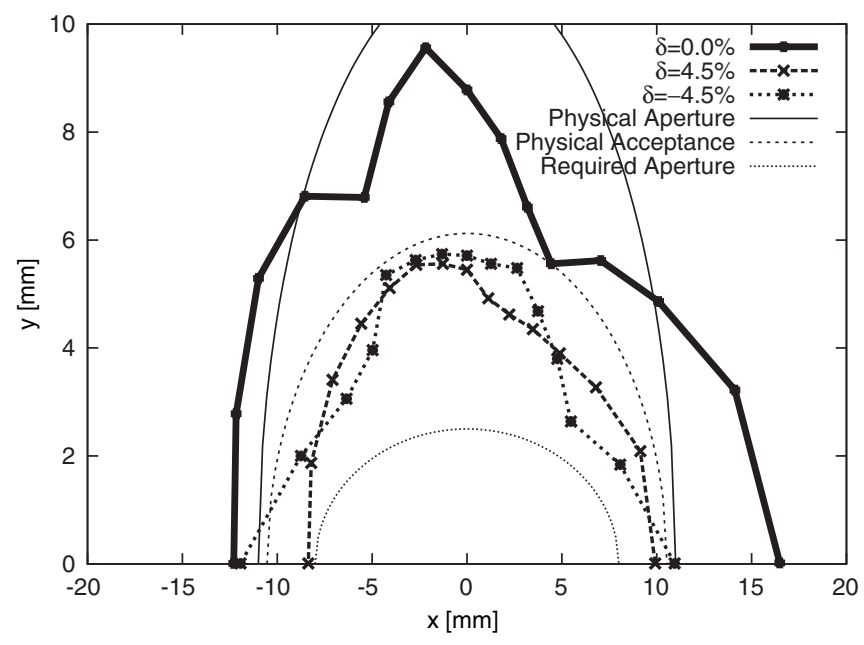

FIG. 8. Dynamic aperture at the center of the $5 \mathrm{~m}$ long straight section for the $3 \mathrm{GeV}$ storage ring with four PMDWs installed. The data results from 6D tracking in TRACY-3 for one synchrotron period $(\approx 500$ turns $)$.

the dynamic aperture was calculated. An example showing the result for 20 error seeds is given in Fig. 9; the applied errors were again $50 \mu \mathrm{m}$ (horizontal and vertical rms misalignment, 2-sigma cutoff) and $0.2 \mathrm{mrad}$ (rms roll error, 2 -sigma cutoff). The reduction of dynamic aperture is clearly visible, however the requirements are still fulfilled. It is known that the tolerances for the girder alignments can be relaxed, if they are tight for the magnets on the girder [25], so in a more realistic study it is expected that $100 \mu \mathrm{m}$ misalignments to girders and $30 \mu \mathrm{m}$ misalignments of the

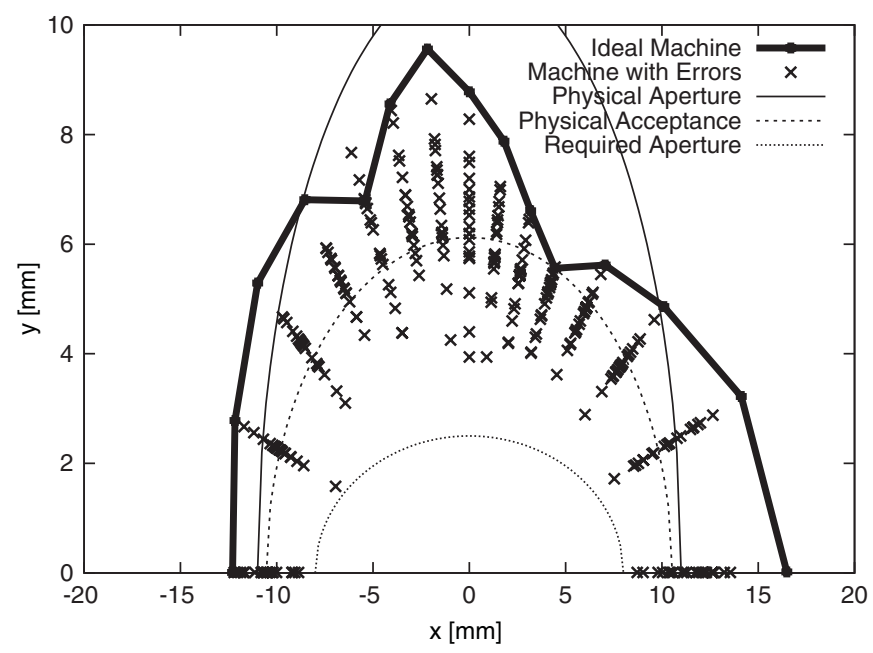

FIG. 9. On-momentum dynamic aperture at the center of the $5 \mathrm{~m}$ long straight section for the $3 \mathrm{GeV}$ storage ring with four PMDWs installed. The crosses show dynamic aperture results for 20 seeds with random error distributions (misalignments and higher-order multipole errors assigned to quadrupoles and sextupoles) and applied orbit correction. While the dynamic aperture is reduced when the errors are included, it still substantially exceeds the requirements.

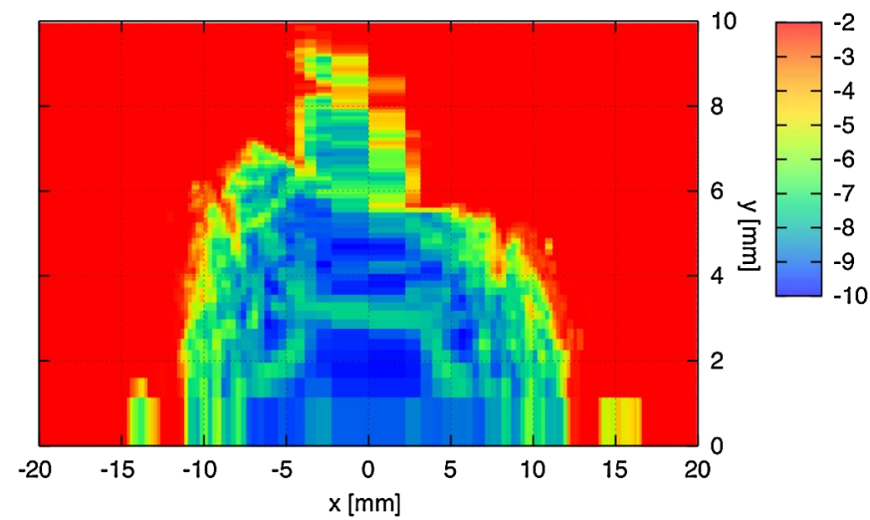

FIG. 10. (Color) Diffusion map (2000 turns) at the center of the $5 \mathrm{~m}$ long straight section for the $3 \mathrm{GeV}$ storage ring with four PMDWs installed. The scale is logarithmic in tune shift from low (blue) to high (red).

magnets with respect to the girder will suffice. For magnets that are machined into a common solid block of iron (see Sec. III A), we expect the alignment of the individual magnets within the same block to be on the order of only $10 \mu \mathrm{m}$.

The dynamic aperture results are confirmed by diffusion maps calculated in TRACY-3. Particles are tracked for a certain number of turns (2048 here) and the final tunes compared with the initial tunes. Diffusion can then be defined as $D=\log \sqrt{\left(\Delta \nu_{x}\right)^{2}+\left(\Delta \nu_{y}\right)^{2}}$ and plotted [37] for various initial transverse coordinates [38]. An example for on-momentum particles taken at the center of the $5 \mathrm{~m}$ long straight section is shown in Fig. 10. The dynamic aperture is essentially the same as in Fig. 8. A stop band (for $\Delta x= \pm 12 \mathrm{~mm}$ caused by the octupolar coupling resonance $2 \nu_{x}-2 \nu_{y}=56$ ) is noticed.

In addition to the diffusion map, frequency map analysis also gives a tune footprint. An example is shown in Fig. 11 where the area within the required dynamic aperture has been inspected for resonant particle loss. Tracking data was gathered by sampling the transverse plane as shown in the diffusion map in the top of Fig. 11. The same data was plotted again in fractional tune space which is shown in the bottom plot. The amplitude-dependent tune shift away from the fractional working point $0.20 / 0.28$ can be clearly recognized. Highest diffusion (roughly $10^{-6}$ ) is encountered as the tune is shifted onto $5 \nu_{x}=211$; this corresponds to the bands seen in the top of Fig. 11 for $|\Delta x| \geq 4 \mathrm{~mm},|\Delta y|>1 \mathrm{~mm}$. Frequency map analysis demonstrates nicely how the amplitude-dependent tune shifts have been adjusted to "wrap around" the working point; tune shifts for betatron motion lead back to the working point thus reducing the overall tune footprint and staying clear of potentially limiting resonances. It has been pointed out that folded regions of phase space are potentially unstable [39]. However, for amplitude excursions within the physical aperture of the MAX IV 


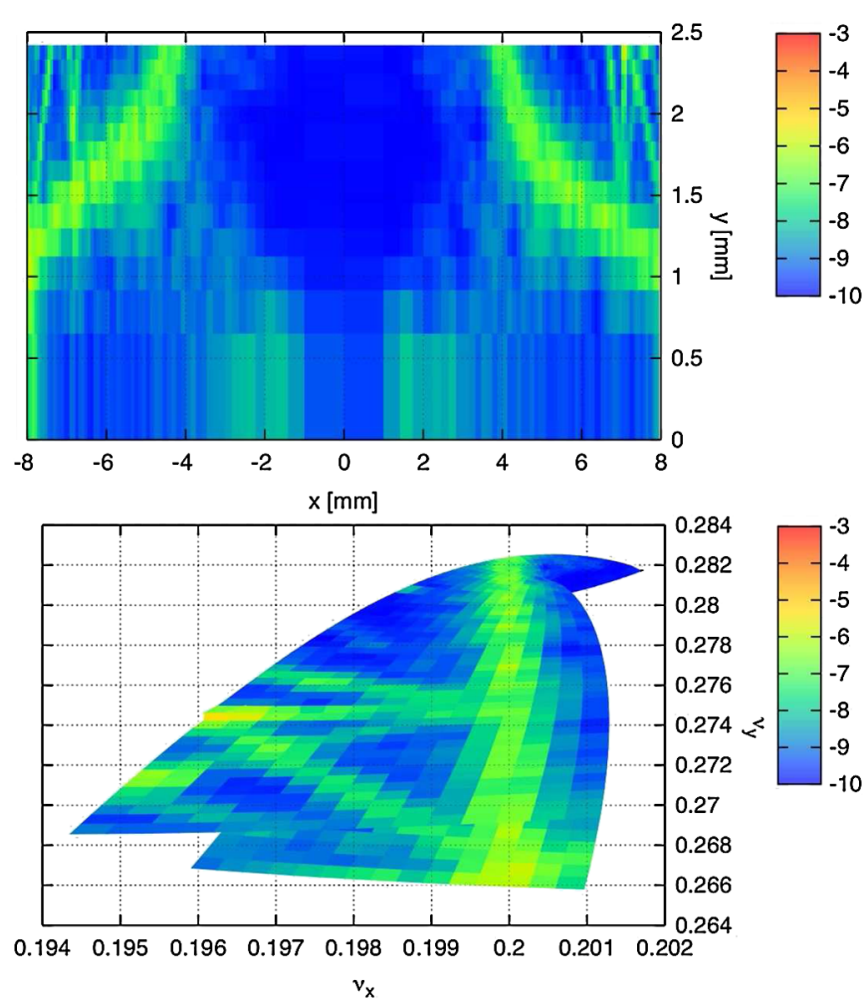

FIG. 11. (Color) Diffusion map (top) and corresponding frequency map (bottom) for on-momentum particles in the $3 \mathrm{GeV}$ storage ring with four PMDWs installed (2000 turns). The scale is logarithmic in tune shift from low (blue) to high (red). Note that compared to the diffusion map given in Fig. 10 the scale here has been shifted to lower diffusion rates by an order of magnitude.

$3 \mathrm{GeV}$ storage ring, the tune shifts have been minimized so that expected tunes stay clear of the actual fold (see Fig. 3). In order to verify that even in the presence of errors the fold does not break down and lead to unstable motion, dynamic aperture was studied when misalignments and multipole errors had been included in the tracking. Although the resulting dynamic aperture is reduced somewhat by these errors (see Fig. 9), it always remains beyond the requirements and no evidence has so far been found that the fold leads to any detrimental effects. The tune footprint confirms that the amplitude-dependent tune shifts in the MAX IV $3 \mathrm{GeV}$ storage ring are very small for on-energy particles (cf. the extremely small area occupied in tune space in Fig. 11) and that there is no resonant particle loss for particles within the dynamic aperture required for injection and the stay-clear area in the straights.

\section{B. Energy acceptance}

In order to achieve the required Touschek lifetime on the order of $10 \mathrm{~h}$, it is crucial to confirm a momentum acceptance of $\pm 4.5 \%$. The overall momentum acceptance is given by the rf momentum acceptance and the lattice momentum acceptance. While choosing a total cavity gap voltage of $1.5 \mathrm{MV}$ or greater is sufficient to achieve $\pm 4.5 \%$ rf momentum acceptance in the storage ring with IDs, it has to be verified that the lattice itself has a momentum acceptance no less than $\pm 4.5 \%$. The small dispersion within the MAX IV $3 \mathrm{GeV}$ achromat ensures that dispersive orbits remain well within the limits of the physical aperture.

Tracking of off-momentum particles was done with TRACY-3. Particles with a constant vertical offset of $\Delta y=$ $+1 \mathrm{~mm}$ were given different momentum offsets and transverse amplitudes. After tracking for 2000 turns (corresponding to roughly a third of damping time) diffusion was calculated. A first example is shown in Fig. 12 where the lattice momentum acceptance can be clearly recognized at $+6 \%$ and $-8 \%$. Particles with energy deviations beyond those limits will be lost regardless of the rf acceptance. Frequency map analysis shows that particle loss is caused by the integer resonance $\nu_{y}=14$ for $\delta>+6 \%$ and by the half-integer resonance $2 \nu_{y}=29$ for $\delta<-8 \%$.

A more detailed view is given in Fig. 13 where horizontal excursions have been limited to low values. This is of interest because the Touschek scattering events leading to large energy deviations take place at the core of the bunch where particle density is greatest. The plot at the top of Fig. 13 is a diffusion map for energy deviations of $\pm 7 \%$ at the bunch core and the bottom plot shows the corresponding frequency map in fractional tune space. Again the "wrap up" of tune shifts around the working point can be recognized: two tune shifts lead away from the fractional working point at $0.20 / 0.28$. Energies above design ultimately lead to lower vertical tunes (covering less than 0.2 in vertical tune); their overall horizontal tune shift is less than 0.03 . The other branch in tune space, leading to larger vertical and lower horizontal tunes, is for particles below design energy; their overall tune variation is however less than $0.1 \times 0.1$. A stop band is recognized for $\delta>$ $+6 \%$ which stems from tune shifts onto the integer reso-

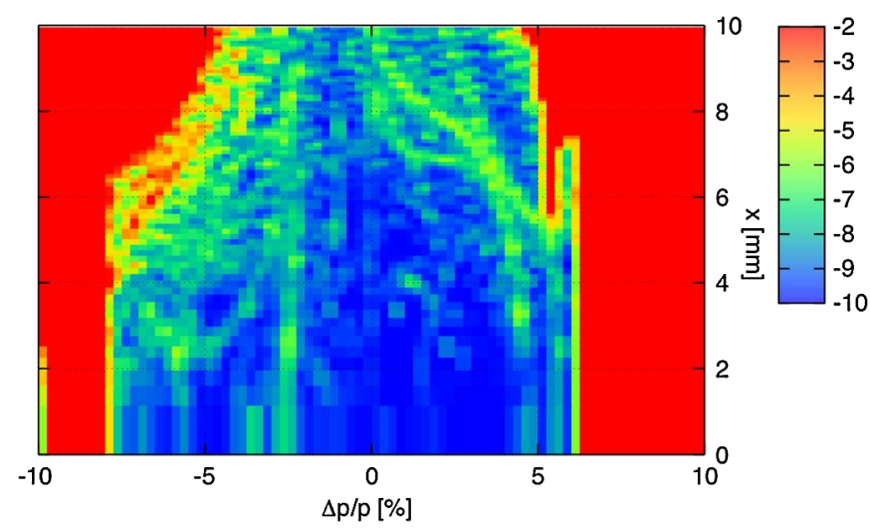

FIG. 12. (Color) Diffusion map taken at the center of the long straight section for off-momentum particles in the $3 \mathrm{GeV}$ storage ring with four PMDWs installed. The scale is logarithmic in tune shift from low (blue) to high (red). 


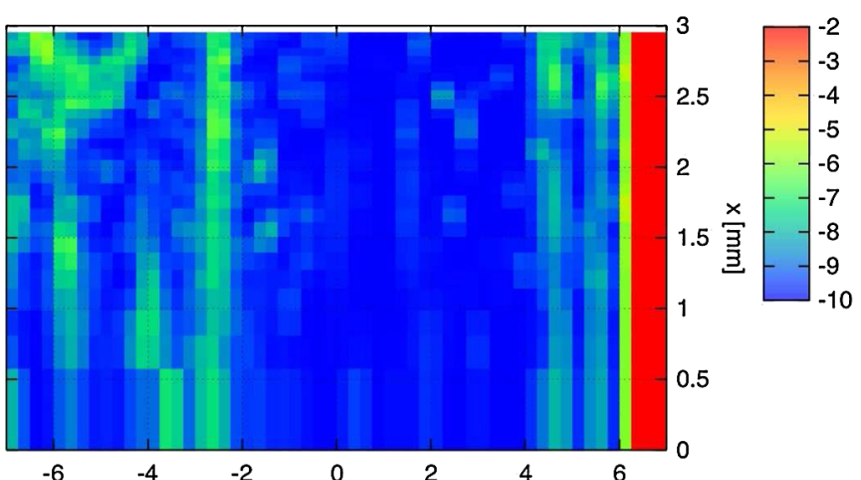

$-6$

$\Delta \mathrm{p} / \mathrm{p}[\%]$

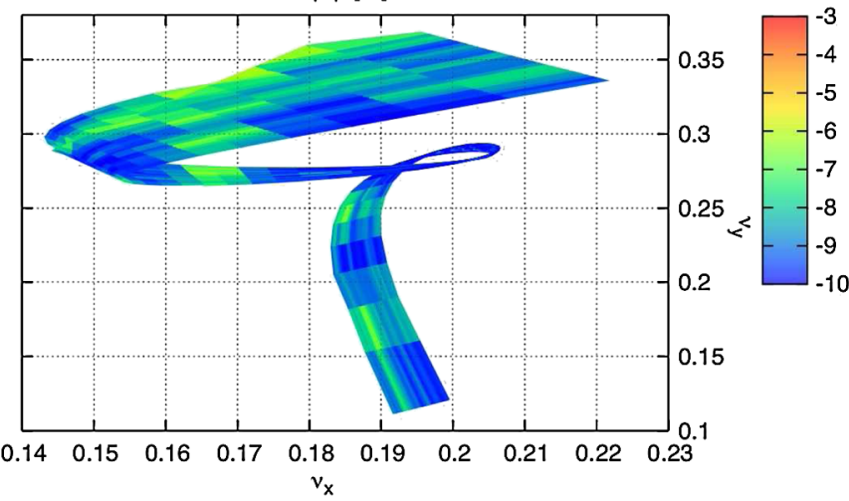

FIG. 13. (Color) Diffusion map (top) and frequency map (bottom) for off-momentum particles in the $3 \mathrm{GeV}$ storage ring with four PMDWs installed. The scale is logarithmic in tune shift from low (blue) to high (red). The linear chromaticity has been adjusted to +1.0 in both planes. The "wrap up" of the chromatic tune shift around the working point is clearly recognized.

nance $\nu_{y}=14$. Particles with energy above design encounter increased diffusion for $\delta \approx+4.75 \%\left(4 \nu_{y}=57\right)$ and $\delta \approx+5.5 \%\left(5 \nu_{y}=71\right)$. For energies below design the main diffusion encountered is due to $6 \nu_{x}=253$ which is seen in the area around $\delta \approx-2.75 \%$ as well as $\delta \approx$ $-6 \%$ for $|\Delta x| \geq 2 \mathrm{~mm}$. In the latter case diffusion is further increased by $3 \nu_{y}=43$.

Frequency map analysis demonstrates that the chromatic tune shifts in the MAX IV $3 \mathrm{GeV}$ storage ring are very small. Horizontal tune excursions remain well below 0.1 for all energies within the rf momentum acceptance; overall vertical excursions are less than 0.3. This shows that even for extreme Touschek events no dangerous low-order resonances will be encountered ensuring good Touschek lifetime and large momentum acceptance.

Since all these considerations have been made for the ideal lattice, the importance of a sophisticated correction scheme has to be emphasized. As has been first demonstrated at the ALS $[40,41]$ and later at several other storage rings, it is indeed possible to meet the design momentum acceptance in the real machine and hence achieve the theoretically predicted Touschek lifetime by careful measurement and correction of the linear [42] and nonlinear [43] optics. For example, meeting the design specifications of the SLS storage ring required the following measures: first, the orbit was corrected, including beam-based BPM calibrations, and the beta functions were restored. Then, small skew quadrupoles located in dispersive and nondispersive sections were employed for precise vertical beam size control, control of vertical dispersion, and suppression of betatron coupling $[44,45]$. Finally, the sextupole pattern was symmetrized by means of small auxiliary sextupoles [46].

\section{Intrabeam scattering}

Because the transverse emittance of the MAX IV $3 \mathrm{GeV}$ storage-ring lattice is so small, intrabeam scattering (IBS) is expected to have a significant influence on the overall equilibrium emittance. Emittance values given so far take into account synchrotron radiation damping and quantum emission fluctuations, but not IBS. In order to quantify the effect of IBS on the equilibrium emittance, calculations have been performed in ZAP [47] and compared with TRACY-3 [48,49]. The ZAP results calculated for a total stored current of $500 \mathrm{~mA}$ and a vertical emittance adjusted to the $1 \AA$ diffraction limit $\varepsilon_{y}=8$ pm rad are summarized in Table V.

For very small lattice emittance values the influence of IBS on equilibrium emittance is very strong. Hence, for the MAX IV $3 \mathrm{GeV}$ bare lattice, the total emittance is significantly underestimated when IBS is not taken into account (the inclusion of IBS leads to a 39\% increase of the equilibrium emittance). However, once the third-harmonic Landau cavities and IDs are added, the additional radiation damping and bunch lengthening strongly reduce the influence of IBS on equilibrium emittance $(<15 \%)$.

\section{Touschek lifetime}

The lattice momentum acceptance has been calculated by tracking with TRACY-3 in 6D including effects of the nonlinear lattice [50]. An error-free machine with realistic vacuum chamber dimensions (horizontal half-aperture $a_{x}=11 \mathrm{~mm}$ ) was used for these calculations. The momentum acceptance for one achromat of the MAX IV

TABLE V. Equilibrium values for the horizontal emittance of the MAX IV $3 \mathrm{GeV}$ storage ring calculated with (right) and without (left) IBS using ZAP. The influence of the third-harmonic Landau cavities (LC) is also shown (with bunch lengthening to $\sigma_{s}=50 \mathrm{~mm}$ assumed).

\begin{tabular}{lcc}
\hline \hline & \multicolumn{2}{c}{$\varepsilon_{x}$ [nm rad] } \\
& Without & With \\
& IBS & IBS \\
\hline Bare lattice & 0.326 & 0.453 \\
Bare lattice with LC & 0.326 & 0.372 \\
Lattice with four PMDWs and LC & 0.263 & 0.297 \\
Lattice with four PMDWs, ten IVUs, and LC & 0.201 & 0.231 \\
\hline \hline
\end{tabular}




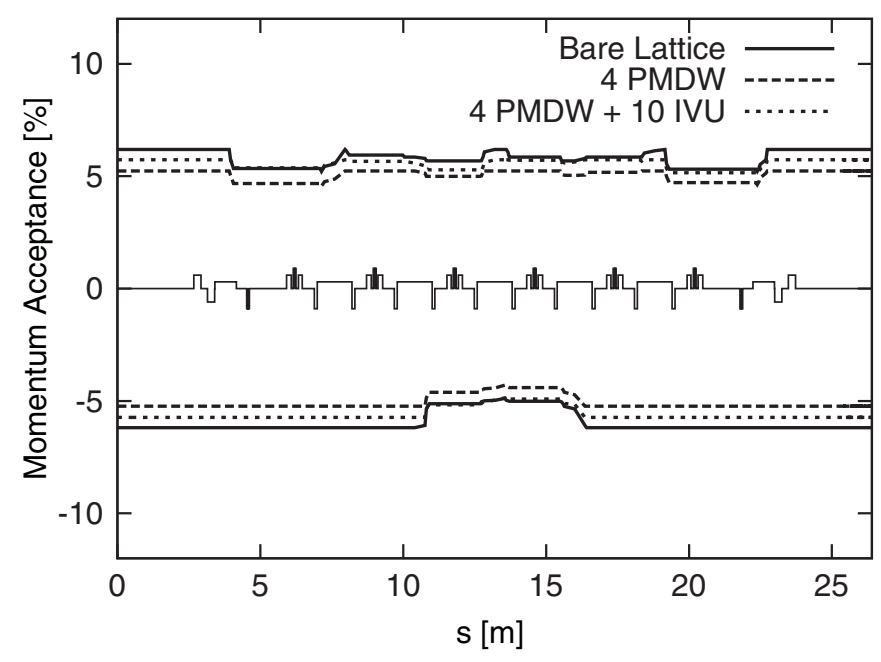

FIG. 14. Momentum acceptance for one achromat of the errorfree MAX IV $3 \mathrm{GeV}$ storage ring calculated with TRACY-3 in 6D. Three configurations of the storage ring are displayed: the bare lattice $\left(V_{\text {cav }}=1.5 \mathrm{MV} \rightarrow \delta_{\text {rf }}=6.2 \%\right)$, a lattice with four PMDWs $\left(V_{\text {cav }}=1.5 \mathrm{MV} \rightarrow \delta_{\text {rf }}=5.3 \%\right)$, as well as a lattice containing four PMDWs and ten IVUs $\left(V_{\text {cav }}=2.0 \mathrm{MV} \rightarrow \delta_{\text {rf }}=\right.$ $5.8 \%$ ). The position of the dipoles, quadrupoles, and sextupoles are indicated at the center.

$3 \mathrm{GeV}$ storage ring in different configurations is shown in Fig. 14. As required by the design specifications, the expected configurations of the MAX IV $3 \mathrm{GeV}$ storage ring always render a lattice momentum acceptance of $4.5 \%$ or higher. For the following considerations on Touschek lifetime we therefore assume an rf momentum acceptance set to $\delta_{\mathrm{rf}}=4.5 \%$ which should then dominate overall momentum acceptance.

Touschek lifetime has been calculated with TRACY-3 [51]. We assumed $500 \mathrm{~mA}$ stored beam current in 176 bunches with a limiting rf acceptance set to $4.5 \%$ as detailed above. A Touschek lifetime of $9.85 \mathrm{~h}$ is obtained with four PMDWs installed and vertical emittance adjusted to the $1 \AA$ diffraction limit $\left(\varepsilon_{y}=8 \mathrm{pmrad}\right)$. For the case where four PMDWs and ten IVUs are installed a Touschek lifetime of $9.31 \mathrm{~h}$ is determined. In addition to the main cavity, we also foresee installation of Landau cavities operating at the third harmonic. The bunch lengthening from the Landau cavities leads to an increase of Touschek lifetime by a factor 4-5. Touschek lifetime for various configurations of the MAX IV $3 \mathrm{GeV}$ storage ring as well as the influence of IBS is shown in Table VI.

It should be pointed out that concerning Touschek lifetime the MAX IV $3 \mathrm{GeV}$ storage ring operates in an atypical regime. As the emittance of the storage ring is further reduced by adding additional IDs, the Touschek lifetime actually improves. This is a unique property among third-generation synchrotron radiation sources and is ultimately a consequence of the ultralow emittance of the MAX IV $3 \mathrm{GeV}$ storage ring. An evaluation of the Touschek function shows that there is a sharp change in
TABLE VI. Touschek lifetime for the MAX IV $3 \mathrm{GeV}$ storage ring calculated with (right) and without (left) IBS using ZAP. The vertical emittance $\varepsilon_{y}=8 \mathrm{pm}$ rad and $\mathrm{rf}$ acceptance $\delta_{\text {rf }}=4.5 \%$ were kept constant. The influence of the Landau cavities (LC) is also shown (with bunch lengthening to $\sigma_{s}=50 \mathrm{~mm}$ assumed).

\begin{tabular}{lcc}
\hline \hline & $\tau_{\text {Touschek }}[\mathrm{h}]$ \\
& Without & With \\
& IBS & IBS \\
\hline Bare lattice & 6.96 & 8.61 \\
Bare lattice with LC & 28.98 & 29.02 \\
Lattice with four PMDWs and LC & 35.57 & 34.52 \\
Lattice with four PMDWs, ten IVUs, and LC & 41.54 & 39.49 \\
\hline \hline
\end{tabular}

behavior once the transverse momenta in the bunch become small compared to the momentum acceptance. The physical explanation is that the transverse momenta in an ultralow emittance bunch are insufficient to generate scattering events which result in momentum deviations that can no longer be contained by the longitudinal acceptance of the machine. This leads to overall fewer Touschek losses and hence increased lifetime at lower emittances. A plot showing Touschek lifetime vs horizontal emittance for the MAX IV $3 \mathrm{GeV}$ storage ring is displayed in Fig. 15. Clearly, further reducing the lattice emittance with additional damping wigglers and insertion devices has a beneficial effect on Touschek lifetime.

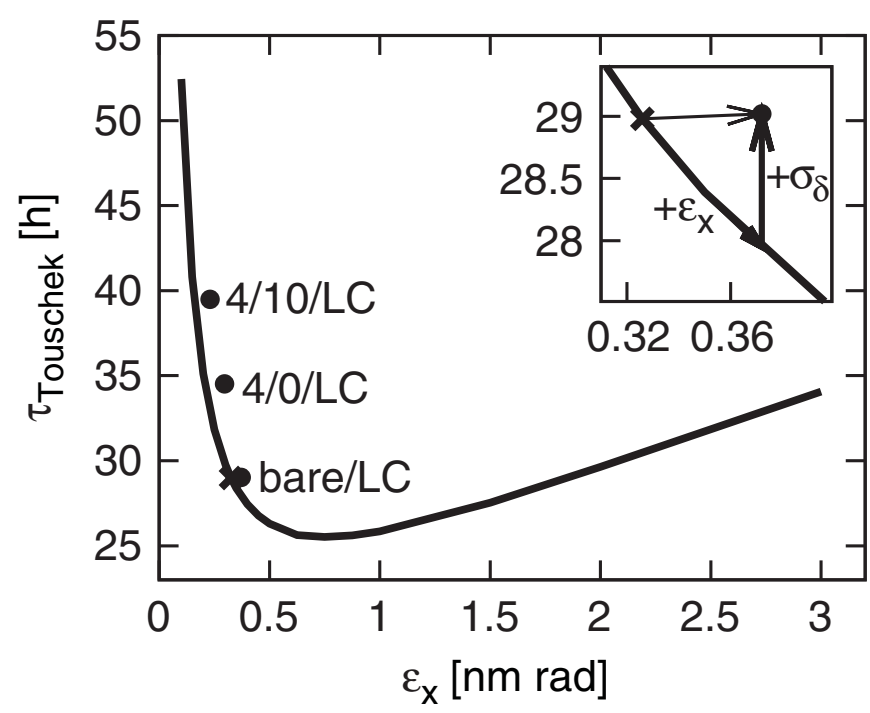

FIG. 15. The trend line shows Touschek lifetime for the bare lattice (IBS neglected) if it were possible to vary the lattice emittance while keeping the energy spread constant. Specific configurations (bare lattice, four PMDWs, and four PMDWs plus ten IVUs; all including LCs) are indicated by crosses and dots. Crosses indicate IBS neglected, dots indicate IBS included. The enlarged segment illustrates the effect of IBS for the bare lattice configuration: while the IBS emittance growth $\left(+\varepsilon_{x}\right)$ leads to a decrease of Touschek lifetime, the IBS energy spread growth $\left(+\sigma_{\delta}\right)$ leads to an increase of Touschek lifetime. 


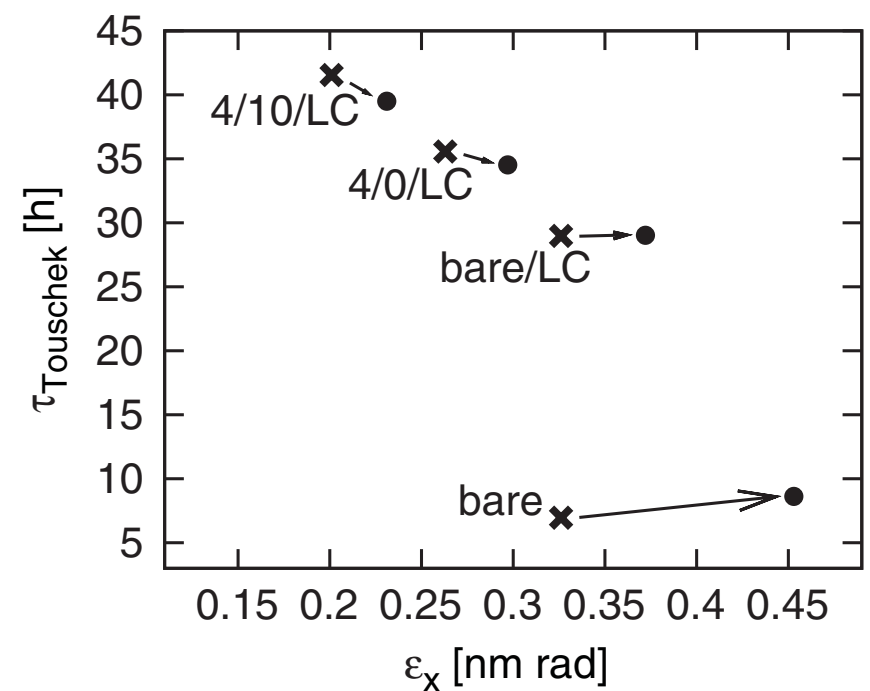

FIG. 16. Touschek lifetime vs horizontal emittance for different configurations of the MAX IV $3 \mathrm{GeV}$ storage ring: bare lattice, lattice with four PMDWs, lattice with four PMDWs and ten IVUs. Where indicated, the effect of the Landau cavities (LC) has been included (with bunch lengthening to $\sigma_{s}=50 \mathrm{~mm}$ assumed). The vertical emittance $\varepsilon_{y}=8 \mathrm{pm}$ rad and $\mathrm{rf}$ acceptance $\delta_{\text {rf }}=4.5 \%$ were kept constant. The effect of IBS is indicated by arrows. Crosses indicate IBS neglected, dots indicate IBS included.

TABLE VII. Contributions to the total MAX IV $3 \mathrm{GeV}$ storage-ring lifetime $\tau$. The results have been calculated for a "worst-case scenario": four PMDWs and ten IVUs are installed in the storage ring while the total applied rf voltage is $1.5 \mathrm{MV}$ which corresponds to an rf acceptance of only $\delta_{\text {rf }}=4.0 \%$.

\begin{tabular}{lc}
\hline \hline & $\tau[\mathrm{h}]$ \\
\hline Elastic gas scattering & 25.4 \\
Inelastic gas scattering & 53.1 \\
Touschek scattering (with Landau cavities) & 25.5 \\
Total & 10.3 \\
\hline \hline
\end{tabular}

With IBS taken into account, one cannot emphasize the benefits of Landau cavities enough. By increasing the bunch length and hence reducing the charge density in the bunch, the Landau cavities not only increase Touschek lifetime, they also reduce the horizontal emittance blowup from IBS, which in the case of the MAX IV
$3 \mathrm{GeV}$ storage ring increases the Touschek lifetime even further. Figures 15 and 16 illustrate this situation.

\section{E. Overall lifetime and top-up injection}

For lifetime and injection considerations we define a "worst-case" scenario. First, the configuration with four PMDWs and ten IVUs ensures large radiated energy losses. Second, we assume no extra rf stations are added; six cavities will give a maximum total cavity voltage of 1.5 MV. This results in a low rf acceptance of $\delta_{\mathrm{rf}}=4.0 \%$. The Touschek lifetime including Landau cavities is then $25.5 \mathrm{~h}$.

The elastic gas scattering lifetime is given by the rest gas composition and the physical acceptance of the machine. A rest gas equivalent of 2 pbar of $\mathrm{CO}$ has been assumed. The vertical acceptance limitation comes from in-vacuum IDs ( $\pm 2 \mathrm{~mm}$ gap, $\beta_{y}=3 \mathrm{~m}$ at the end of the ID), whereas the horizontal acceptance is given by the maximum of the horizontal beta function $\left(\beta_{\mathrm{x}, \max }=10 \mathrm{~m}\right.$, chamber inner radius $\pm 11 \mathrm{~mm}$ ). This gives an elastic scattering lifetime of $25.4 \mathrm{~h}$. The inelastic gas scattering lifetime also depends weakly on momentum acceptance. Assuming the same rest gas pressure, the inelastic gas scattering lifetime is calculated as $53.1 \mathrm{~h}$ for an energy acceptance of $4.0 \%$. These lifetime contributions are summarized for the "worstcase" scenario in Table VII. When the MAX IV $3 \mathrm{GeV}$ storage ring is operated with IDs and Landau cavities, as a consequence of the long Touschek lifetime, overall beam lifetime will no longer be Touschek dominated.

It is foreseen to have top-up injection into the MAX IV $3 \mathrm{GeV}$ storage ring. Assuming a maximum current variation of $0.2 \%$ is tolerated and taking into account the calculated "worst-case" total lifetime of $10.3 \mathrm{~h}$, an injection of $1.8 \mathrm{nC}$ will be required every $74 \mathrm{sec}$. The MAX IV $3 \mathrm{GeV}$ injector linac will be capable of delivering up to $10 \mathrm{nC}$ in a $100 \mathrm{~ns}$ shot. Therefore the required top-up injection into the storage ring can be performed with a single shot. If we require five damping times for the residual kicker bump excitation of the stored beam to damp down, this yields a user dead time of $0.06 \%$. A variable multishot injection at reduced charge is presently being considered in order to implement a filling pattern feedback. This would however come at the expense of more frequent top-up injections or a longer injection dead time (dominated by the repetition rate of the injector rather than the damping time) [16].

TABLE VIII. Insertion devices for the MAX IV $3 \mathrm{GeV}$ storage ring. The peak magnetic field $\hat{B}$, device length $L$, and period length $\lambda$ are given.

\begin{tabular}{lccc}
\hline \hline & $\hat{B}[\mathrm{~T}]$ & $\lambda[\mathrm{mm}]$ & $L[\mathrm{~m}]$ \\
\hline Permanent-magnet damping wiggler (PMDW) & 2.22 & 80 & 2.0 \\
In-vacuum permanent-magnet undulator (PMU) & 1.28 & 19.0 & 3.0 \\
Cryogenically cooled in-vacuum undulator (CPMU) & 1.38 & 17.5 & 3.0 \\
Superconducting undulator (SCU) & 1.70 & 14.0 & 3.0 \\
\hline \hline
\end{tabular}




\section{F. Brightness}

The IDs for the MAX IV $3 \mathrm{GeV}$ storage ring will be tailored to the specific needs of the beam lines. Conventional planar undulators as well as elliptically polarizing undulators will be installed in addition to the previously mentioned damping wigglers. The most demanding undulators are the flat field undulators for the production of undulator radiation at the highest possible photon energy, where the undulators combine shortest possible period length with highest possible magnetic peak field. The combination of short period length and high magnetic peak field points towards narrow-gap invacuum undulators of hybrid type, where blocks of permanent-magnet material are combined with soft magnetic materials. The peak field of an in-vacuum undulator can be increased by lowering the temperature of the magnetic material and recently cryogenically cooled invacuum undulators have been developed $[52,53]$.

Superconducting undulators would, provided that the technical problems of building superconducting undulators can be overcome, outperform undulators based on permanent-magnet technology. The MAX IV $3 \mathrm{GeV}$ storage ring is well suited for the installation of superconducting undulators because of the long bunch length and soft ends of the dipoles flanking the straight section, which give a moderate heat load to the cryostat of a superconducting undulator. Table VIII shows different types of IDs under consideration for the MAX IV $3 \mathrm{GeV}$ storage ring. The undulators have been optimized for short period length while retaining overlap between first and third harmonic and have a maximum $K$ value of $\approx 2.2$.

Figure 17 shows the expected brilliance at peak energy of the undulators in Table VIII installed at the MAX IV $3 \mathrm{GeV}$ storage ring. The brilliance has been calculated with the code SPECTRA [54] using a horizontal emittance of $0.26 \mathrm{~nm}$ rad, a vertical emittance of $8 \mathrm{pm} \mathrm{rad}$, and an rms

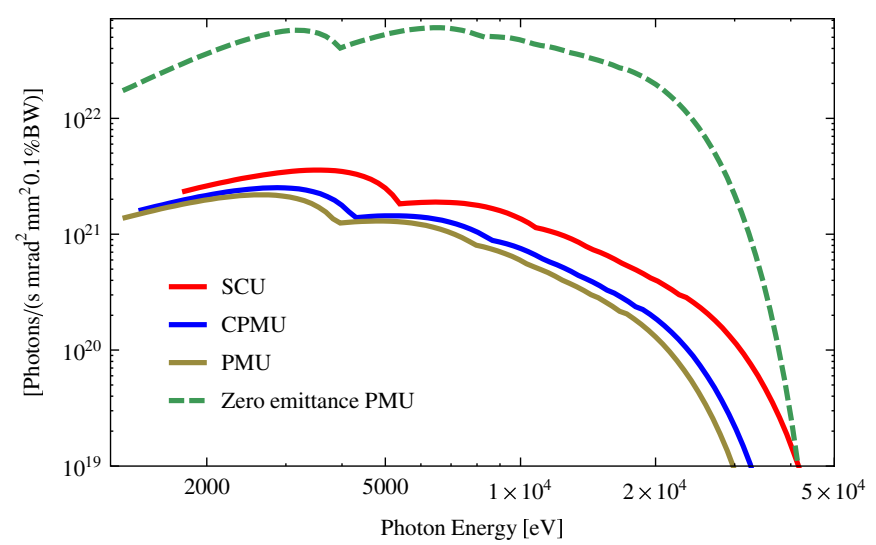

FIG. 17. (Color) Brilliance limit for the MAX IV $3 \mathrm{GeV}$ storage ring for in-vacuum permanent-magnet undulators (PMU), cryogenically cooled in-vacuum undulators (CPMU), and superconducting undulators (SCU). The undulator parameters are given in Table VIII. energy spread of $0.1 \%$. As a comparison, the brilliance of the PMU installed in the "ultimate source" (zero horizontal and vertical emittance, but with the same energy spread) has been included in Fig. 17. The plotted values are brilliance at peak energy and not maximum brilliance, which is found at slightly lower photon energy.

\section{CONCLUSIONS AND OUTLOOK}

The MAX IV $3 \mathrm{GeV}$ storage ring has been shown to be a feasible concept to generate high-brightness $\mathrm{x}$-ray radiation. The unprecedented ultralow emittance is made possible by a novel lattice design approach using compact multibend achromats with gradient dipole magnets. This low emittance is then further reduced by the introduction of strong damping wigglers and in-vacuum undulators. The required strong focusing, however, puts high demands on magnet design and vacuum system. Problems associated with vacuum pressure and transverse instability are mitigated by the use of NEG coating and a $100 \mathrm{MHz}$ main rf system. If construction proceeds as expected, the MAX IV $3 \mathrm{GeV}$ storage ring should go into operation in 2015 . With a constant stored current of $500 \mathrm{~mA}$ and a horizontal emittance below $0.3 \mathrm{~nm}$ rad, it will become the brightest storage ring-based synchrotron radiation source worldwide.

Emphasis is now being put on several remaining issues. Full 6D tracking for a lattice with realistic multipole field errors and magnet misalignments should give a better understanding of the dynamic behavior in the real machine. The orbit correction scheme is also under investigation: it is foreseen to implement correctors as additional windings in the sextupole magnets and install the BPMs between these sextupoles and the adjacent quadrupoles. We plan to verify that a fast orbit correction scheme with this corrector/BPM layout fulfills the tight beam stability requirements (due to the ultralow beam emittance an RMS beam stability in the long straight section on the order of $300 \mathrm{~nm}$ is required). Vibration studies with the foreseen low-height massive concrete support system are under way. Finally, the design of IDs and tailoring of IDs to specific beam line requirements is an ongoing activity.

[1] M. Böge, in Proceedings of the 8th European Particle Accelerator Conference, Paris, 2002 (EPS-IGA and CERN, Geneva, 2002), pp. 39-43.

[2] A. Nadji et al., in Proceedings of the 2007 Particle Accelerator Conference, Albuquerque, New Mexico, 2007 (IEEE, Albuquerque, New Mexico, 2007), pp. 932-934.

[3] R. Bartolini, in Proceedings of the 2007 Particle Accelerator Conference, Albuquerque, New Mexico, 2007 (Ref. [2]), pp. 1109-1111.

[4] NSLS-II Preliminary Design Report, http://www.bnl.gov/ nsls2/project/PDR/. 
[5] S. Krinsky, J. Bengtsson, and S. Kramer, in Proceedings of the 10th European Particle Accelerator Conference, Edinburgh, Scotland, 2006 (EPS-AG, Edinburgh, Scotland, 2006), pp. 3487-3489.

[6] PETRA III Technical Design Report, http://petra3.desy.de/ general/tdr/.

[7] MAX IV Conceptual Design Report, http://www.maxlab. lu.se/maxlab/max4/index.html.

[8] S. Werin, S. Thorin, M. Eriksson, and J. Larsson, Nucl. Instrum. Methods Phys. Res., Sect. A 601, 98 (2009).

[9] M. Sjöström, H. Tarawneh, E. Wallén, and M. Eriksson, Nucl. Instrum. Methods Phys. Res., Sect. A 577, 425 (2007).

[10] M. Sjöström, E. Wallén, M. Eriksson, and L. J. Lindgren, Nucl. Instrum. Methods Phys. Res., Sect. A 601, 229 (2009).

[11] M. Eriksson, L. J. Lindgren, M. Sjöström, E. Wallén, L. Rivkin, and A. Streun, Nucl. Instrum. Methods Phys. Res., Sect. A 587, 221 (2008).

[12] M. Eriksson, Å. Andersson, S. Biedron, M. Demirkan, G. Leblanc, L. Lindgren, L. Malmgren, H. Tarawneh, E. Wallén, and S. Werin, in Proceedings of the 8th European Particle Accelerator Conference, Paris, 2002 (Ref. [1]), pp. 686-687.

[13] H. Tarawneh, M. Eriksson, L. J. Lindgren, and B. Anderberg, Nucl. Instrum. Methods Phys. Res., Sect. A 508, 480 (2003).

[14] M. Eriksson et al., in Proceedings of the 2007 Particle Accelerator Conference, Albuquerque, New Mexico, 2007 (Ref. [2]), pp. 74-76.

[15] M. Eriksson, A. Hansson, S. C. Leemann, L.-J. Lindgren, M. Sjöström, E. Wallén, L. Rivkin, and A. Streun, in Proceedings of the 11th European Particle Accelerator Conference, Genoa, 2008 (EPS-AG, Genoa, Italy, 2008), pp. 2007-2009.

[16] MAX IV Detailed Design Report, http://www.maxlab. lu.se/maxlab/max4/index.html.

[17] S. Lee and L. Teng, in Proceedings of PAC, San Francisco, CA, 1991 (IEEE, New York, 1991), pp. 2679-2681.

[18] Dispersion in the short straights is very low so that installing the rf cavities here will prevent emittance dilution from synchrobetatron oscillations.

[19] The focusing quadrupoles between the dipoles have actually been split up and separated to make room for a short focusing sextupole in between, see Sec. II B.

[20] This is a requirement in order to make installation of invacuum superconducting IDs feasible.

[21] C. Steier, D. Robin, A. Wolski, G. Portmann, and J. Safranek, in Proceedings of the 20th Particle Accelerator Conference, Portland, OR, 2003 (IEEE, New York, 2003), pp. 3213-3215.

[22] OPA Lattice Design Code, http://slsbd.psi.ch/ streun/opa/.

[23] Although this appears to be a very low setting for the corrected chromaticity, it is considered feasible here because of the low accelerating radio frequency (see Sec. III C).

[24] J. Bengtsson, SLS Internal Report No. 9/97, http:// slsbd.psi.ch/pub/slsnotes/sls0997.pdf.

[25] J. Bengtsson, W. Joho, P. Marchand, G. Muelhaupt, L. Rivkin, and A. Streun, Nucl. Instrum. Methods Phys. Res., Sect. A 404, 237 (1998).
[26] J. Bengtsson, Tracy-2 User's Manual (unpublished).

[27] M. Cornacchia and Y. Chin, Part. Accel. 17, 191 (1985).

[28] S. C. Leemann and A. Streun (unpublished).

[29] M. Sjöström, E. Wallén, M. Eriksson, and L.-J. Lindgren, Nucl. Instrum. Methods Phys. Res., Sect. A 597, 170 (2008).

[30] R. Kersevan, M. Hahn, and I. Parat, in Proceedings of the 10th European Particle Accelerator Conference, Edinburgh, Scotland, 2006 (Ref. [5]), pp. 1420-1422.

[31] E. Wallén, M. Berglund, H. Svensson, and M. Eriksson, in Proceedings of the 17th International Vacuum Congress (IVC-17), Stockholm, Sweden, 2007 [J. Phys. Conf. Ser. 100, 092019 (2008)].

[32] A. Hansson, M. Berglund, and E. Wallén, in Proceedings of the 11th European Particle Accelerator Conference, Genoa, 2008 (Ref. [15]), pp. 3693-3695.

[33] E. Al-Dmour, in Proceedings of the 11th European Particle Accelerator Conference, Genoa, 2008 (Ref. [15]), pp. 31-35.

[34] A. Andersson, M. Bergqvist, M. Eriksson, L. Malmgren, and L. Thånell, in Proceedings of the 8th European Particle Accelerator Conference, Paris, 2002 (Ref. [1]), pp. 2118-2120.

[35] An option to use eight such cavities if the ring is fully loaded with IDs is being investigated. The total gap voltage of $2 \mathrm{MV}$ would still give a maximum rf momentum acceptance of $5.3 \%$ for the fully loaded machine.

[36] P. Elleaume, in Proceedings of the 3rd European Particle Accelerator Conference (EPAC-92), Berlin, Germany, 1992 (Editions Frontières, Gif-sur-Yvette, France, 1992), pp. 661-663.

[37] For reasons of clarity, the plots displayed here show a slightly modified diffusion defined as $D=\min [-2$, $\left.\log \sqrt{\left(\Delta \nu_{x}\right)^{2}+\left(\Delta \nu_{y}\right)^{2}}\right]$.

[38] L. Nadolski and J. Laskar, Phys. Rev. ST Accel. Beams 6, 114801 (2003).

[39] J. Laskar, in Proceedings of the 20th Particle Accelerator Conference, Portland, OR, 2003 (Ref. [21]), pp. 378-382.

[40] D. Robin, J. Safranek, and W. Decking, Phys. Rev. ST Accel. Beams 2, 044001 (1999).

[41] C. Steier, D. Robin, L. Nadolski, W. Decking, Y. Wu, and J. Laskar, Phys. Rev. E 65, 056506 (2002).

[42] J. Safranek, Nucl. Instrum. Methods Phys. Res., Sect. A 388, 27 (1997).

[43] R. Bartolini, I. P. S. Martin, J. H. Rowland, P. Kuske, and F. Schmidt, Phys. Rev. ST Accel. Beams 11, 104002 (2008).

[44] A. Andersson, M. Böge, A. Lüdeke, V. Schlott, and A. Streun, Nucl. Instrum. Methods Phys. Res., Sect. A 591, 437 (2008).

[45] Å. Andersson, M. Böge, A. Lüdeke, and A. Streun, in Proceedings of the 11th European Particle Accelerator Conference, Genoa, 2008 (Ref. [15]), pp. 1983-1985.

[46] M. Böge, A. Lüdeke, and A. Streun, in Proceedings of PAC09, Vancouver, Canada, 2009, http://www.triumf.info/ hosted/PAC09/.

[47] M. S. Zisman, S. Chattopadhyay, and J. J. Bisognano, ZAP User's Guide, LBL 21270 (December 1986).

[48] J. Le Duff, Report No. CERN 89-01.

[49] V. Litvinenko (private communication).

[50] TRACY-3 also includes the effects of nonlinear momentum 
compaction. However, we note that the nonlinear contribution to momentum compaction in the MAX IV $3 \mathrm{GeV}$ storage ring is very weak because our multibend achromat gives comparable linear and second-order coefficients: $\alpha_{c}=3.1 \times 10^{-4}$ and $\alpha_{2}=1.3 \times 10^{-4}$. Even for a particle at the limit of the rf acceptance $(\delta=5 \%)$, the secondorder contribution to momentum compaction amounts to only $2 \%$ of the linear contribution.

[51] A. Streun, SLS Internal Report No. 18/97, http://slsbd.psi. ch/pub/slsnotes/sls1897a.pdf.
[52] J. Chavanne, M. Hahn, R. Kersevan, C. Kitegi, C. Penel, and F. Revol, in Proceedings of the 11th European Particle Accelerator Conference, Genoa, 2008 (Ref. [15]), pp. 2243-2245.

[53] T. Hara, T. Tanaka, H. Kitamura, T. Bizen, X. Maréchal, T. Seike, T. Kohda, and Y. Matsuura, Phys. Rev. ST Accel. Beams 7, 050702 (2004).

[54] T. Tanaka and H. Kitamura, J. Synchrotron Radiat. 8, 1221 (2001). 